\title{
Investigations on diurnal and seasonal variations of Schumann resonance intensities in the auroral region
}

\author{
Claudia Rossi, Paolo Palangio and Franco Rispoli \\ Istituto Nazionale di Geofisica e Vulcanologia, Osservatorio Geofisico di L'Aquila, Italy
}

\begin{abstract}
Measurements of the magnetic component of the Schumann resonance in the frequency range 6-14 Hz were performed at high latitude location (TNB Antarctica; geographic coordinates: $74.7^{\circ} \mathrm{S}, 164.1^{\circ} \mathrm{E}$; geomagnetic coordinates: $80.0^{\circ} \mathrm{S}, 307.7^{\circ} \mathrm{E}$; $\mathrm{LT}=\mathrm{UT}+13$; $\mathrm{MLT}=\mathrm{UT}-8$; altitude $=28 \mathrm{~m}$ a.s.l.), during the two years 1996-1997. TNB is a particularly important observation site located in a region characterised by a high electromagnetic activity in the ELF and VLF bands. Moreover its remote location in Antarctica provides the important advantage that electromagnetic background noise is not corrupted by anthropogenic noise and that the continental lightning activity is very low. The combination of low additional anthropogenic electromagnetic radiation and low atmospheric noise in this area allows detailed investigations into wave generation and amplification in the polar ionosphere and magnetosphere not possible anywhere else in the world. This paper reports the study of the magnetic power of the $8 \mathrm{~Hz}$ Schumann resonance mode. For both the years considered diurnal and long-term seasonal variations were observed.
\end{abstract}

Key words resonances - ELF observations - ionospheres

\section{Introduction}

Schumann resonance oscillations are the most remarkable natural electromagnetic phenomena in the lower frequency side of ELF range. As pointed out by several studies (Raemer, 1961; Balser and Wagner, 1962a; Pierce, 1963; Ogawa et al., 1969; Polk, 1969; Galejs, 1972; Clayton and Polk, 1977; Sentman, 1996; Nickolaenko, 2002) the intensities of the Schumann resonances reflect the totality of global thunderstorm activity exciting the zeroth-order Transverse Magnetic (TM0) normal modes of the Earth-iono-

Mailing address: Dr. Claudia Rossi, Istituto Nazionale di Geofisica e Vulcanologia, Osservatorio Geofisico di L'Aquila, Castello Cinquecentesco, Via Castello, 67100 L'Aquila, Italy; e-mail: rossi@ingv.it sphere cavity. Moreover the excitation of the Earth-ionosphere cavity can be the effect of hydromagnetic waves propagation through the ionosphere (Abbas, 1968; Nickolaenko, 2002).

The space separating the Earth and the ionosphere indeed forms a cavity, which can support electromagnetic standing waves with wavelengths comparable to planetary dimensions. Large electromagnetic transients, such as lightning, radiate broadband electromagnetic impulses that spread radially into the cavity. The low frequency impulse components can circumnavigate the globe several times before suffering serious degradation, and so produce a resonant line spectrum, by the phase addition and cancellation of waves that have traversed the global circumference several times along multiple paths. The resulting waves are quasi-transverse electromagnetic (quasi-TEM) normal modes of the Earthionosphere cavity. The total resonant spectrum is the incoherent superposition of the effects from the totality of global lightning.

These resonances, called Schumann resonances, can, in principle, be detected from any 
place on the planet, and, away from thunderstorms and artificial electromagnetic noise sources, constitute the principal component of the natural background of the electromagnetic spectrum over the frequency range $6-50 \mathrm{~Hz}$.

Resonance proprieties of the Earth-ionosphere cavity were first predicted and discussed theoretically by Schumann (1952) and the earliest experimental detection of the resonances was made by Schumann and König (1954). The first spectral representation showing the resonance lines was presented by Balser and Wagner (1960). During the five years following these initial results, experimental measurements of increasing sophistication were performed (Raemer, 1961; Balser and Wagner, 1962a,b, 1964; Pierce, 1963; Rycroft, 1965). At the same time several theoretical descriptions of the resonances were advanced to take into account realistic proprieties of the ionosphere (Wait, 1992).

Following these initial reports, there were about two decades of active research on the entire subject of ELF propagation. Although study of the Schumann resonances formed a part of this research, the primary emphasis in these studies was on frequencies above $45 \mathrm{~Hz}$. The general electromagnetic theory for VLF and ELF waves in the Earth-ionosphere system is contained in the books by Galejs (1972), Bliokh et al. (1980), and Polk (1982).

Several subsequent research and theoretical studies have also be important to the Schumann resonance description (Nickolaenko and Rabinovich, 1982; Sentman, 1987, 1990, 1996; Sentman and Fraser, 1991; Burke and Jones, 1992; Wait, 1992; Füllekrug, 1995; Füllekrug and FraserSmith, 1996; Märcz, 1997; Morente et al., 2003).

The most recent comprehensive reference works on Schumann resonances are the books by Sentman (1995), and Nickolaenko (2003).

Many published works show that the Schumann resonance intensities undergo a diurnal modulation in both the vertical electric field and horizontal magnetic field intensities at particular locations (Keefe et al., 1964; Polk, 1969; Clayton, 1977; Sentman and Fraiser, 1991; Sátori and Zieger, 1996; Märcz et al., 1997).

Keefe et al. (1964) and Sentman and Fraiser (1991) have shown that there may be a local time effect in the diurnal intensity variation. In particular they observed that the Schumann resonance intensities maximize near local noon at each location.

Märcz et al. (1997) measurements have evidenced annual and seasonal variations in the relative amplitudes of Schumann resonances' vertical electric component in the first three modes (Meloni et al., 1992).

The diurnal and seasonal variations of the background noise may be the result of changes in both the propagation conditions (frequency and amplitude response of the Earth-ionosphere cavity), and the source characteristics (intensity and distribution of thunderstorm). In particular one of the most important features in the propagation of lightning signals may be the variation of the ionosphere morphology from day to night. The dayside ionosphere indeed is denser and lower compared to the nightside. The TNB station is far from lightning sources, so we expect that the main contribution to background noise variation at this location is the ionospheric one.

This paper presents the diurnal variation of the magnetic component of the $8 \mathrm{~Hz}$ Schumann resonance for the 1996 and the 1997 years. Section 2 describes the instrumentation and the observation site. Data analysis is described in Section 3 , where we show that two principal peaks are present in the diurnal variation and we illustrate their seasonal variation.

\section{Instrumentation}

Within the framework of the ITALIANTARTIDE project, continuous geomagnetic measurements were performed in the frequency range $5-11 \mathrm{~Hz}$ during 1996-1997 at TNB Antarctica (TNB Antarctica; geographic coordinates: $74.7^{\circ} \mathrm{S}, 164.1^{\circ} \mathrm{E}$; geomagnetic coordinates: $80.0^{\circ} \mathrm{S}, 307.7^{\circ} \mathrm{E} ; \mathrm{LT}=$ $=\mathrm{UT}+13 ; \mathrm{MLT}=\mathrm{UT}-8$; altitude $=28 \mathrm{~m}$ a.s.l. $)$. The TNB observation site is located in a region characterised by a high electromagnetic activity in the ELF and VLF bands. TNB indeed lie in the auroral region, which is connected by lines of force that reach the magnetopause in the daylight side and the plasmasheet of the magnetotail in the nightside. This consents to look extreme regions of the magnetosphere. Moreover its remote loca- 
tion in Antarctica provides the important advantage that electromagnetic background noise is not corrupted by anthropogenic noise an that the continental lightning activity is very low. The combination of low additional anthropogenic electromagnetic radiation and low atmospheric noise in this area allows detailed investigations on the wave generation and amplification in polar ionosphere and magnetosphere not possible anywhere else in the world.

The observations reported in this work were derived from data obtained from a wide band ELF monitoring. The measuring equipment includes a three search coil sensors, which were

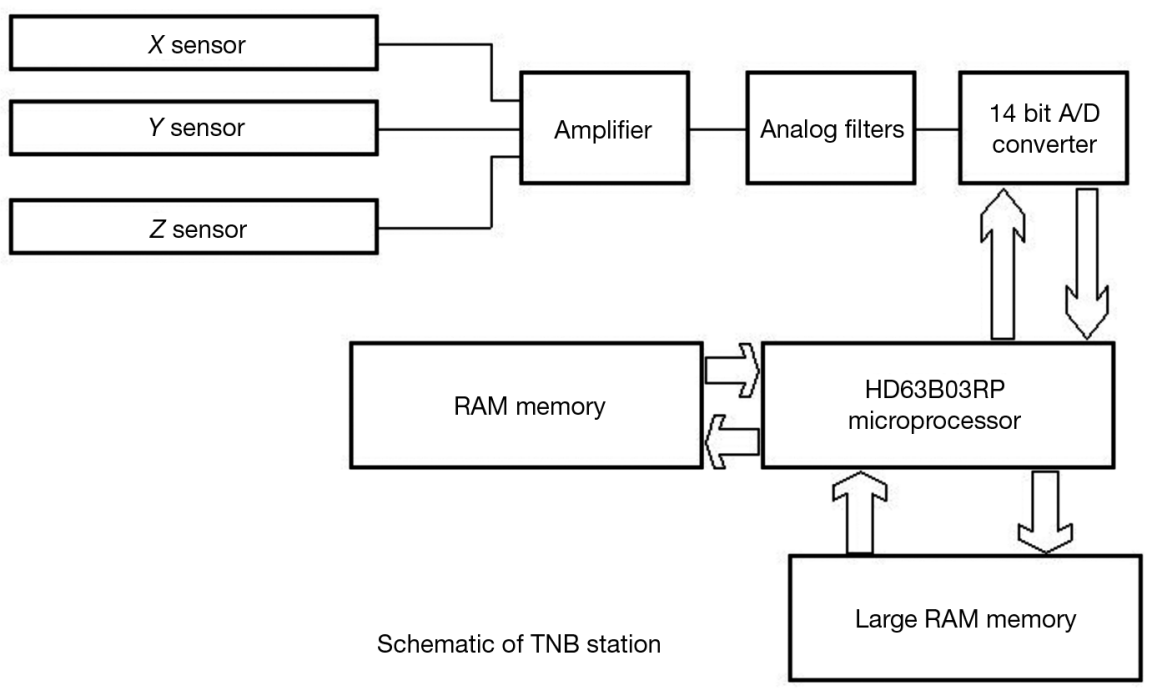

Fig. 1. Block diagrams of the whole TNB station: $X, Y$, and $Z$ signals were processed, and then stored in a large memory.
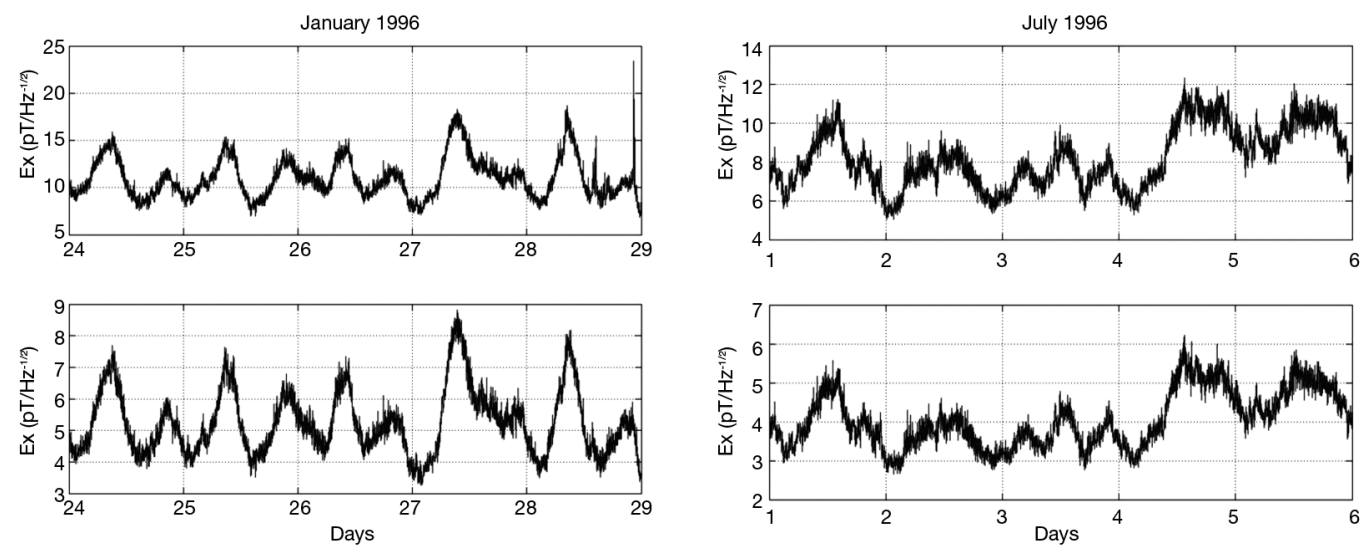

Fig. 2. Magnetic power of the $8 \mathrm{~Hz}$ Schumann resonance mode over the two time interval January 24-29, 1996, and July 1-6, 1996. Marked diurnal variations, characterised by two principal peaks are evident. The two peaks profiles seem to vary from day-to-day. 
used in order to obtain measurements of the directional characteristics of the noise (Palangio, 1993). A single sensor is composed of 8 coils of 50000 turns each (fig. 1). Two of the sensors were oriented in the horizontal plane, one in the magnetic north-south $(X)$ and the other in the magnetic east-west $(Y)$, the third is oriented in the local vertical direction $(Z)$. The magnetic declination mean value at the time of measurements was $137^{\circ}$.

The station is powered by battery and solar cells, and includes a microprocessor, filters and data collecting system. Data recording electronics consists of a microcomputer interface, which performs sampling, a/d conversion, and storing of $X, Y$ and $Z$ signal amplitudes in a small memory. At the end of each measurement the data stored in the interface memory were transferred into the microcomputer for processing. The results were stored in large memory (fig. 1). The station is therefore able to record data for long time in autonomous way. A block diagram of the whole experimental system is presented in fig. 1 .

The station can perform wide band geomagnetic measurements from $0.0005 \mathrm{~Hz}$ to $1000 \mathrm{~Hz}$. To obtain the measurements considered in the present paper, signals were sampled at a frequency of $32 \mathrm{~Hz}$, and then double integrated in the frequency range $f=f_{0} \pm \Delta f / 2$ (where $f_{0}=8 \mathrm{~Hz}$ and $\Delta f=6 \mathrm{~Hz}$ ), and in a time window of $120 \mathrm{~s}$.

The measurements are expressed in spectral density unit $\left(f T^{2} / \mathrm{Hz}\right)$.

\section{Data analysis}

To motivate the analysis procedure we show in fig. 2 the plot of the magnetic power the horizontal components of the $8 \mathrm{~Hz}$ Schumann resonance mode versus the Local Time (LT), for two time intervals 5 days long, selected respectively in January and July 1996. For each day two principal peaks are evident: a first one (M1) lying in the time interval 8-12 LT, and a second one (M2) approximately at 20-21 LT. The two peak profile varies from day-to-day. We performed a data analysis to study the seasonal variation of the hour of appearance and amplitude of the two peaks. For each day we computed hourly means (LT) of the magnetic power. Then we determined peaks time positions and amplitudes using a program based on the derivative method.

\subsection{M1 peak annual variation}

Figure 3 shows the hour of occurrence (LT) of the M1 peak with respect to the day for the horizontal components of the $8 \mathrm{~Hz}$ Schumann magnetic power in the 1996 and 1997 years. In order to reduce day-to-day fluctuations, M1 hourly position values were averaged over 15 days. Observed trends are similar for the two years. For both the $X$ and $Y$ components M1 peak appear at about 8.30 LT at the end of January (Austral summer), it moves toward the noon from January to June, and it appear at $\sim 12 \div 12.40$ about the Austral winter solstice. Then it moves back to reach again its position at about 8.30 LT in November.

The M1 peak intensity seasonal variation for 1996 and 1997 is shown in fig. 4. Intensity values are averaged over 15 days. In the 1996 trend three different regions are visible for both the $X$ and $Y$ components:

- In the first region, extending from the end of January to about the 20th April, the intensity decrease from its maximum value to its minimum value. The decrease trend is compatible with the linearity.

- In the second region extending from about the 20th April to about the 10th August, the intensity grows, reach a relative maximum and then decrease to its minimum.

- In the third region extending from about the 10th August to the end of October, the intensity grows from its minimum value to its maximum value.

The 1997 intensity trend is quite similar to the 1996 one, but the second region is anticipated by about 50 days (fig. 4).

\subsection{M2 peak annual variation}

The seasonal variation of the M2 peak hour of occurrence (LT) is shown in fig. 5. M2 peak values were averaged over 15 days to reduce statistical fluctuations. The M2 peak time of occurrence does not seem to vary in valuable 

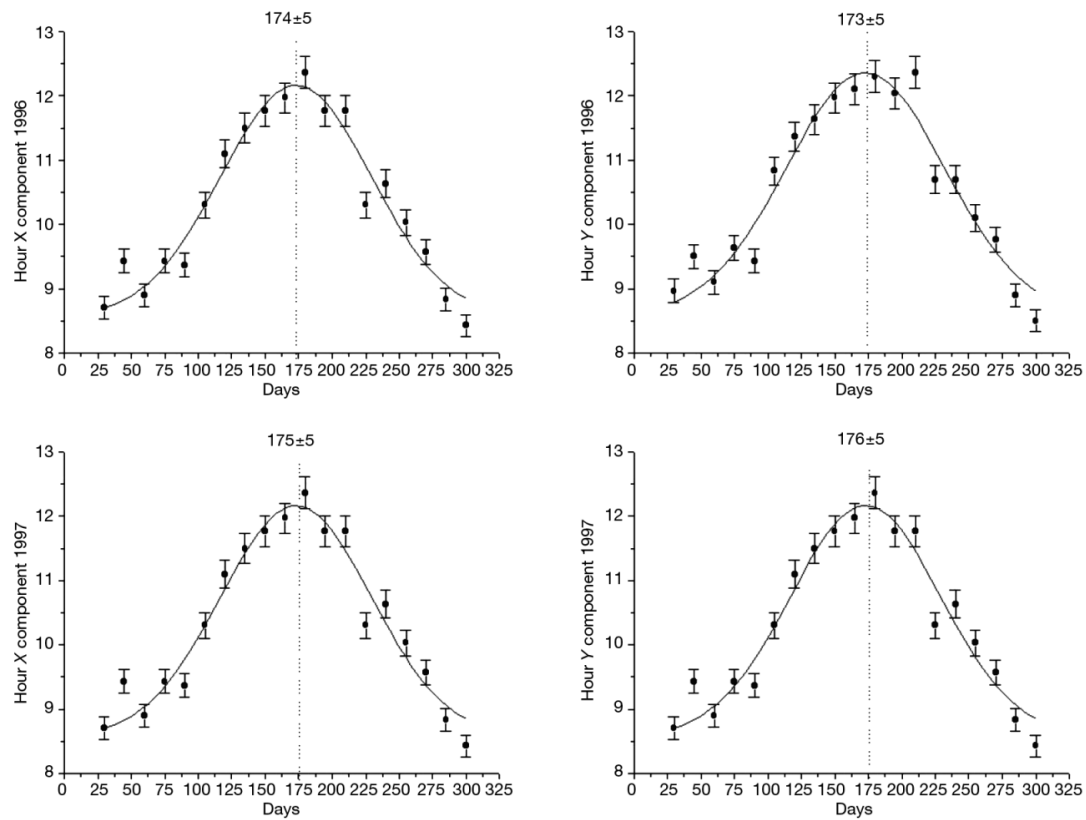

Fig. 3. Plot of the hour of occurrence (LT) of the M1 maximum versus the day for the horizontal components of the magnetic power of the $8 \mathrm{~Hz}$ Schumann resonance mode in the years 1996-1997. M1 appear early in the morning $(\sim 8.30 \mathrm{LT})$ in the austral summer and lightly after the noon $(12.30 \div 13.00)$ about the austral winter solstice.
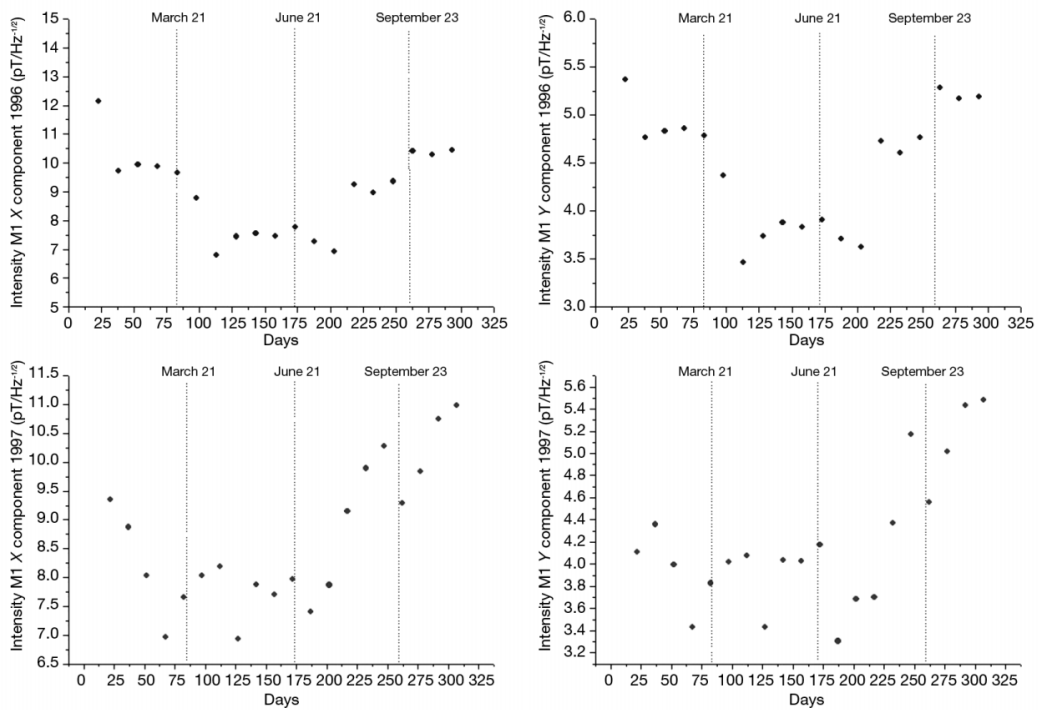

Fig. 4. Plot of the intensity of the M1 peak versus the day for the horizontal components of the magnetic power of the $8 \mathrm{~Hz}$ Schumann resonance mode in the years 1996-1997. Equinoxes and austral winter solstice are showed. In 1996 the intensity is maximum in austral summer, and reach a relative maximum approximately in correspondence of the winter solstice. In 1997 the relative maximum is anticipated by about 50 days. 

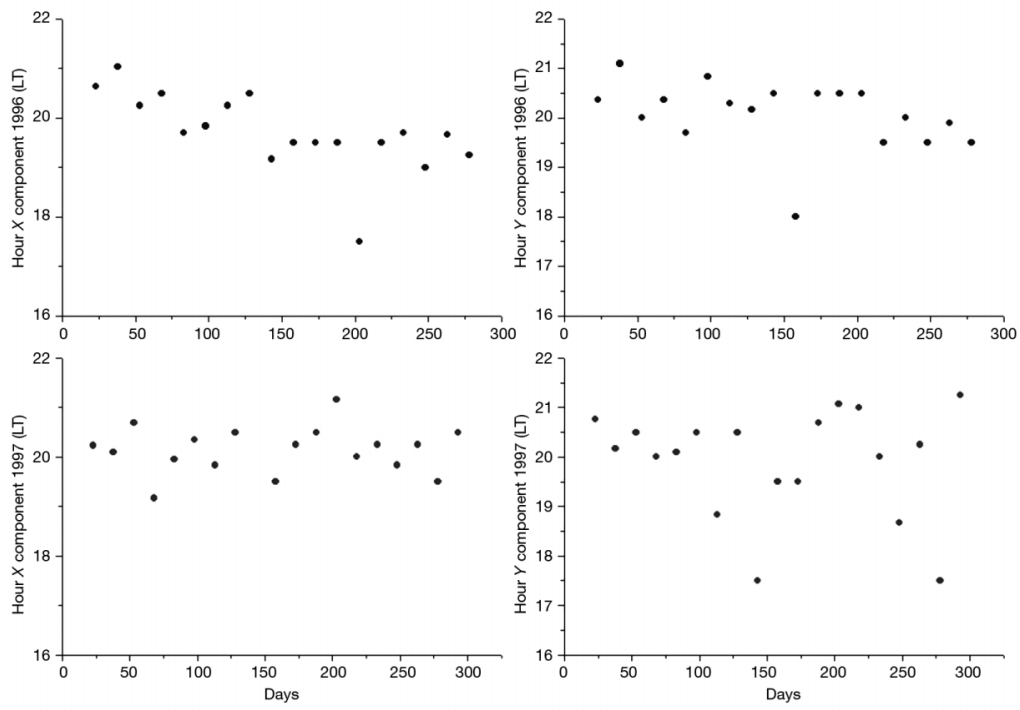

Fig. 5. Plot of the hour of occurrence (LT) of the M2 maximum versus the day for the horizontal components of the magnetic power of the $8 \mathrm{~Hz}$ Schumann resonance mode in the years 1996-1997. M2 peak position does not show a significant seasonal variation.
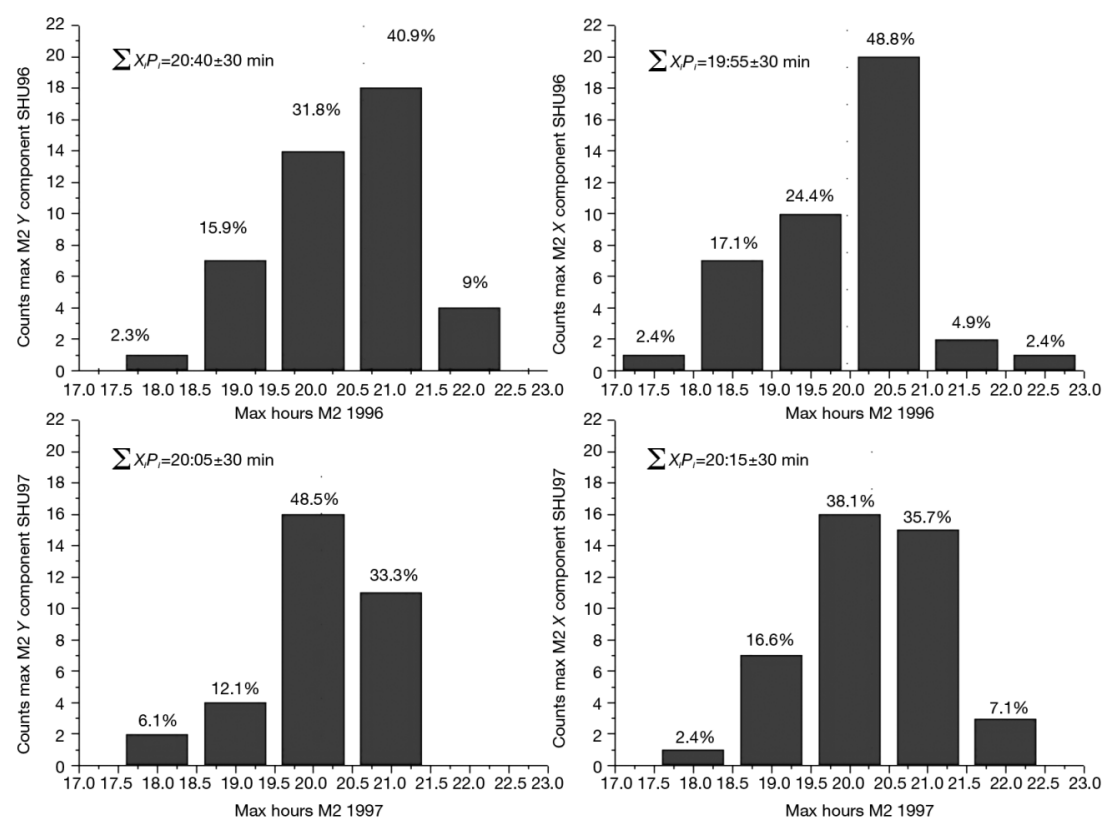

Fig. 6. Statistical distribution of the M2 peak position with respect to the hour (LT). Computed weighted mean values are also shown. 

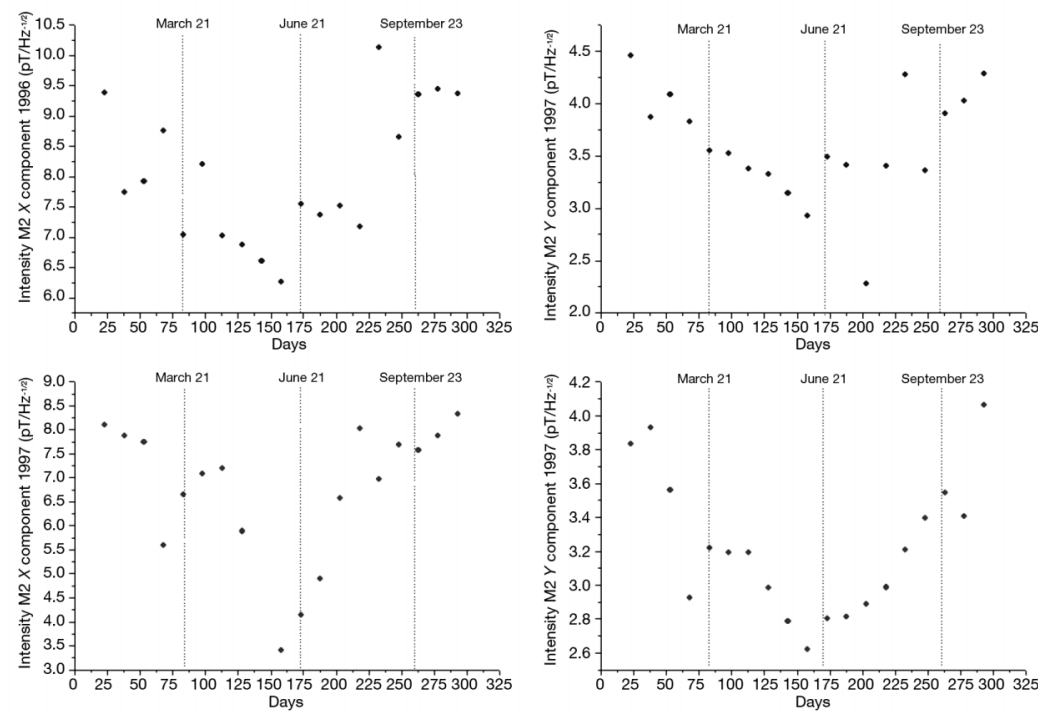

Fig. 7. Plot of the intensity of the M2 peak versus the day for the horizontal components of the magnetic power of the $8 \mathrm{~Hz}$ Schumann resonance mode in the years 1996-1997. Equinoxes and austral winter solstice are showed. The intensity is maximum in austral summer, and minimum in austral winter.

way during the year. Figure 6 shows the statistical distributions of the M2 hour of occurrence, and the computed weighted mean values are reported.

The M2 peak intensity as a function of the day is shown in fig. 7. The M2 intensity decreases from January (austral summer) to June (austral winter), and grows from August to October.

As outlined before, the M2 peak analysis is complicated because M2 is missing in some days. The work to explain this phenomenology is still in progress.

\subsection{Polarization analysis}

The statistical analysis of the polarization indicate that during quiet geomagnetic conditions the waves are always elliptically polarized, the ellipticities falling mainly in the range from 0.1 to 0.3 . Preliminary results from this analysis show also that sometimes the waves are linearly polarised, the degree of polarization of the signals is equal to 1 , the field components are mutually coherent and the wave field is po- larized in a single plane. This is consistent with the presence of a single plane wave in a single wave mode. The occurrence frequency of this event appears to increase with increasing ULF activity, it is predominantly a dayside phenomenon with a broad maximum within four hours of local noon (MLT). The duration of this events varies approximately from 50 to $1000 \mathrm{~s}$. We have looked at a few individual cases, and conducted a superposed epoch analysis to determine the average behavior for all the events. The results are shown in fig. 8. As can be seen, there are five plots: the coherency, the degree of polarization, the angle of polarization, the ellipticity and the sense of polarization. The ellipticity of the magnetic field polarization is estimated from the eigenvector associated to the nonnull eigenvalue of the magnetic spectral matrix. The ellipticity is near zero which implies a linear polarization. The determination of the sense of polarization in the plane perpendicular to $Z$ is based on the sign of the imaginary part of the cross-spectrum between the $X$ and $Y$ components. Figure 9a shows the spectral shape of linearly polarized signals, as can be seen the shape 

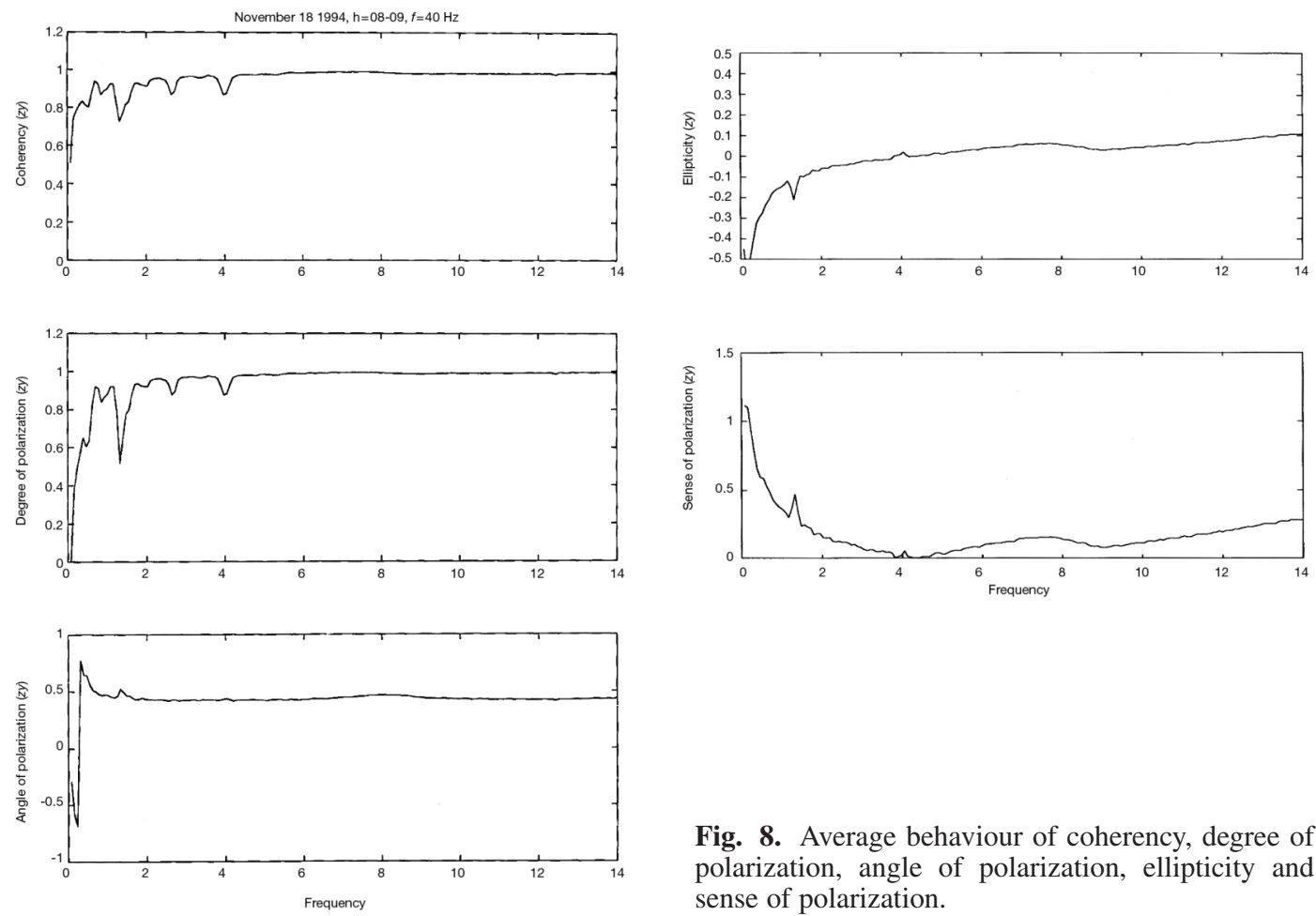

Fig. 8. Average behaviour of coherency, degree of polarization, angle of polarization, ellipticity and sense of polarization.
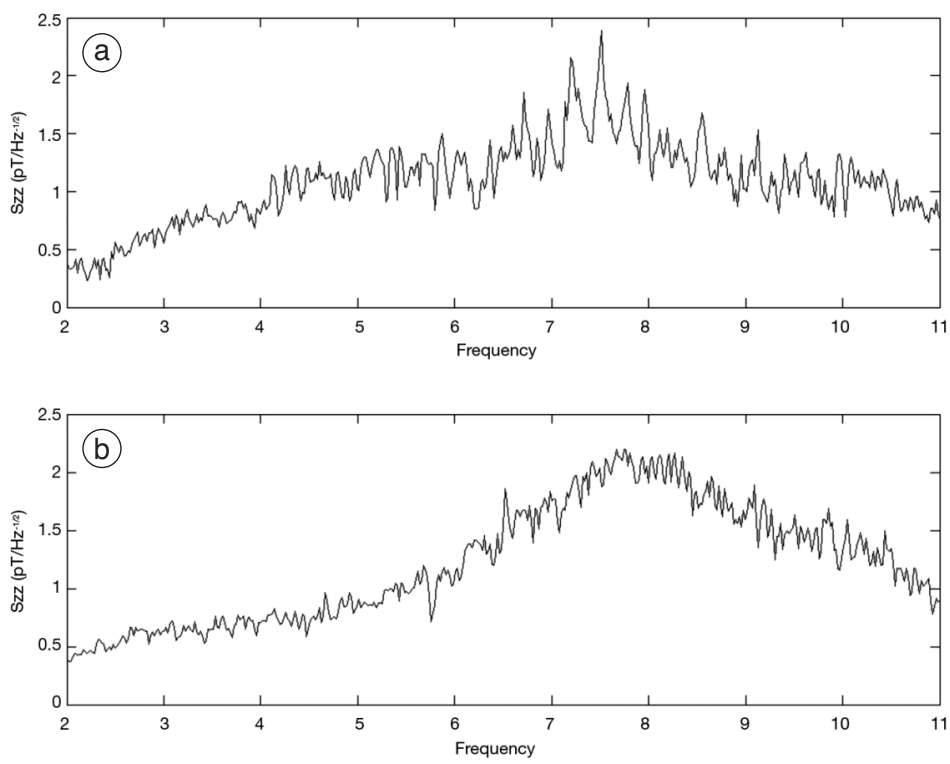

Fig. 9a,b. Spectral shape of a) linearly polarized signals, b) signals due to the traditional generation mechanism. 
is much harper than that appearing in fig. $9 \mathrm{~b}$ which represents the spectral power density of the signal due to the traditional generation mechanism. The shape of the spectra is strongly influenced by the number of averaged FFT used to get the spectral analysis. This influence is, however, not of great practical importance since we compare values obtained by the same procedure.

\subsection{Discussion}

The analysis performed has evidenced an annual variation in the M1 peakhour of appearance. In their works Keefe et al. (1964), and Sentman and Fraiser (1991) observed that the intensities of the horizontal components of the Schumann resonances maximize near local noon at the locations of their measurements (Rhode Island, Germany, California and Western Australia). They attributed the observed modulation to the variation of the height of the $D$-region of the ionosphere.

Due to the high latitude of our station, a direct comparison with our measurements is quite difficult. We think that plasma structures in the $F$ layer may play a role as wave-guides, which could canalise the $S R$ waves along the geomagnetic field. For frequencies in the $S R$ band the wavelengths are comparable with the thickness of the ionosphere, and the waves could be confined into the region around the Alfven minimum. Such structures above the Polar Regions enhance the probability that the magnetospheric signals exit from the Earth-ionosphere waveguide. Satellites measurements have shown that low frequency waves are generated in the magnetosphere by different types of instability, and propagate along the lines of force of the geomagnetic field to reach the lower ionosphere in the polar regions where signals from low and middle latitude strokes are weak. Moreover the observations from satellites show ELF emissions at high latitudes, which maximize in the interval of Magnetic Latitude (ML) $73^{\circ}-80^{\circ}$. Cerenkov radiation involving a longitudinal resonance and low energy electrons connected to physical processes in the magnetosphere might produce them.
Waves linearly polarized could originate in the magnetosphere, when observed from the ground, they appear to originate from currents in the ionosphere. The signals observed on the ground can be interpreted as a convolution of a source function, the intensity of the waves in the magnetosphere, and the propagation function, which takes account of the effects of ionospheric conductivity inhomogeneities on the mode waves as they travel through the ionosphere, guided along the magnetic field.

The intensity of both M1 and M2 peaks undergoes an annual variation characterised by a maximum value in the Austral summer. Sátori and Zieger (1996), and Märcz et al. (1997), performed measurements of the vertical electric field component in the frequency range of Schumann resonance at Nagycenk Observatory in Hungary. They found an annual variation in the vertical electric field intensity. In particular they observed a maximum in the Northern Hemisphere summer. Märcz et al. (1997) correlated the Schumann resonances amplitudes to simultaneous measurements of the atmospheric electric potential gradient. They explained their results in terms of correlation between surface air temperature and parameters of the atmospheric electric global circuit. In particular the observed summer maximum in the Schumann resonances amplitudes is considered partly a response to the intrinsic global source (i.e. to the increased global thunderstorm activity in this season), and partly the response to the source proximity effect.

Our observations agree with those of Sátori and Zieger (1996), and Märcz et al. (1997), however, due to the TNB location, they are not affected by source proximity effect.

In future extended statistical analysis we intend to study the possible connection between Pc3-4 pulsations and events linearly polarized, because most of this signals were observed with magnetic pulsations in the Pc3-4 range.

\section{Summary}

Measurements of the magnetic power of the $8 \mathrm{~Hz}$ SR mode were performed at TNB during the years 1996-1997. Diurnal and seasonal vari- 
ability were observed in the horizontal components of the magnetic power. In the diurnal trend two principal peaks were found: a first one (M1) lying in the time interval 8-12 LT, and a second one (M2) approximately at 20-21 LT. For the M1 peak we observe a seasonal variation in both hours of appearance and amplitude. The study of the M2 peak is more difficult, because M2 is missing on some days. We notice a seasonal variation of its amplitude, while its hour of appearance does not seem to vary during the year.

The observed ELF in the auroral zone is different from that at middle or low latitudes. We think that plasma structures in the $F$ layer may play a role as wave-guides, which could canalise the SR waves along the geomagnetic field. This is compatible with satellite measurements. Moreover in the auroral regions the $F$ layer density has a maximum in the hours of the M2 peak appearance.

This preliminary analysis represents a reliable starting point for further studies to make use of long data series to identify a possible magnetosphere-ionosphere influence superimposed on the Earth-ionosphere cavity mode. These studies will be the subject of a separate paper.

\section{REFERENCES}

ABbas, M. (1968): Hydromagnetic wave propagation and excitation of Schumann resonances, Planet. Space Sci., 16, 831-844.

BALSER, M. and C.A. WAGNER (1960): Observations of Earthionosphere cavity resonances, Nature, $\mathbf{1 8 8}$, p. 638.

BALser, M. and C.A. Wagner (1962a): Diurnal power variations in the Earth-ionosphere cavity modes and their relationship to world-wide thunderstorm activity, $J$. Geophys. Res., 67, p. 619.

BALsER, M. and C.A. WAGNER (1962b): On frequency variations of the Earth-ionosphere cavity modes, J. Geophys. Res., 67, p. 4081.

BALSER, M. and C.A. WAGNER (1964): Thunderstorm excitation of the Earth-ionosphere cavity, in Propagation of Radio Waves at Frequencies Below $300 \mathrm{kc} / \mathrm{s}$, edited by W.T. BlackBand (Pergamon Press), pp. 257.

Bliokh, P.V., A.P. NikOlaENKo and YU.F. FiliPPOV (1980): Schumann Resonances in the Earth-Ionosphere Cavity (Peter Perigrinus, London), pp. 168.

BuRKE, C.P. and D.L. JONES (1992): An experimental investigation of ELF attenuation rates in the Earth-ionosphere duct, J. Atmos. Terr. Phys., 54, 243-250.

Clayton, M.D. and C. Polk (1977): Diurnal variation and absolute intensity of world-wide lightning activity, September 1970 to May 1971, in Electrical Processes in Atmospheres, edited by H. DOLEZALEK and R. REITER (Verlag, Darmstadt, Germany), p. 440.

FÜllEKRUG, M. (1995): Schumann resonances in magnetic field components, J. Atmos. Terr. Phys., 57 (5), 479-484.

FülleKrUG, M. and A.C. FRASER-SMith (1996): Further evidence for a global correlation of the Earth-ionosphere cavity resonances, Geophys. Res. Lett., 23 (20), 27732776.

GaleJs, J. (1972): Terrestrial Propagation of Long Electromagnetic Waves (Pergamon, Tarrytown, New York).

KeEFE, T.J., C. PolK and H. KÖNIG (1964): Results of simultaneous ELF measurements at Branneburg (Germany) and Kingston, RI, in NBS Report on Symposium on Ultra Low Frequency Electromagnetic Fields, Boulder, Colorado (National Bureau of Standards, Gaithersburg, Md., Aug.), Contrib. 12, pp. 12-1 to 12-14.

MÄrCZ, F., G. SÁtori and B. ZIEGER (1997): Variations in Schumann resonances and their relation to atmospheric electric parameters at Nagycenk station, Ann. Geophysicae, 15 (12), 1604-1614.

Meloni, A., P. Palangio and A.C. Fraser Smith (1992): Some characteristics of the ELF/VLF radio noise measured near L'Aquila, Italy, IEEE Trans. Ant. Prop., 40 (2), 233-236.

Morente, J.A., G.J. Molina-Cuberos, J.A. Portí, B.P. BesSer, A. Salinas, K. Schwingenschuch and H. LichteNEGGER (2003): A numerical simulation of Earth's electromagnetic cavity with the Transmission Line Matrix method: Schumann resonances, J. Geophys. Res., 108 (A5), 1195, doi: 10.1029/2002JA009779.

NickolaEnKo, A.P. and M. HayakaWA (2002): Resonances in the Earth-Ionosphere Cavity (Kluwer, Dordrecht), pp. 392.

NickolaenKo, A.P. and L.M. Rabinovich (1982): Possible global electromagnetic resonances on the planets of the solar system, Cosmic Res., 20, 67-71.

OgaWA, T., Y. TANAKA and M. YASUHARA (1969): Schumann resonances and worldwide thunderstorm activity, in Planetary Electrodynamics, edited by S. CoRONITI and J. HuGHES (Gordon and Breach, New York), vol. 2, 85-91.

Palangio, P. (1993): Radioricezione ELF-VLF, Ann. Geofis., XXXVI (5-6), 99-114.

PIERCE, E.T. (1963): Excitation of Earth-ionosphere resonances by lightning flashes, J. Geophys. Res., 68, 4125.

PoLK, C. (1969): Relation of ELF noise and Schumann resonances to thunderstorm activity, in Planetary Electrodynamics, edited by S. Coroniti and J. HugHes (Gordon and Breach, New York), vol. 2, 55-83.

RAEMER, E.T. (1961): On the extra low frequency spectrum of the Earth-ionosphere cavity response to electrical storms, J. Geophys. Res., 66, p. 1580.

SchumanN, W.O. (1952): Über die strahlungslosen Eigenschwingungen einer leitenden Kugel, die von einer Luftschicht und einer Ionosphärenhülle umgeben ist, Zeitschrift für Naturforschung, 7a, p. 149.

SCHUMANN, W.O. and H. KÖNIG (1954): Über die Beobactung von Atmospherics bei geringsten Frequenzen, Naturwiss, 41, 183-184.

SENTMAN, D.D. (1987): Magnetic elliptical polarization of Schumann resonances, Radio Sci., 22, 595-606.

SENTMAN, D.D. (1990): Approximate Schumann resonance parameters for a two-scale-height ionosphere, J. Atmos. Terr. Phys., 52, 35-46. 
SENTMAN, D.D. (1996): Schumann resonance spectra in a two-scale-height Earth-ionosphere cavity, J. Geophys. Res., 101 (D5), 9479-9487.

SENTMAN, D.D. and B.J. Fraser (1991): Simultaneous observations of Schumann resonances in California and Australia: evidence for intensity modulation by the local height of the D region, J. Geophys. Res., 96, 15973-15984. WAIT, J.R. (1992): On ELF transmission in the Earth-ionosphere waveguide, J. Atmos. Terr. Phys., 54, 109-111.

(received December 18, 2006; accepted April 16, 2007) 